\title{
Prácticas, conocimientos y percepciones que dificultan la conservación de la agrobiodiversidad. El caso del cinturón hortícola platense, Argentina*
}

María Luz Blandi**

Manhattan Sioux Cavalcante***

Natalia Agustina Gargoloff ****

Santiago Javier Sarandón*****

doi: doi:10.11144/Javeriana.cdri3-78.pcpd

Recibido: 20I6-07-08 Aprobado: 20I6-06-I2 Disponible en línea: 20I6-I2- 20

Cómo citar este artículo: Blandi, M., Cavalcante, M., Gargoloff, N. y Sarandón, S.(2016). Prácticas, conocimientos y percepciones que dificultan la conservación de la agrobiodiversidad. El caso del cinturón hortícola platense, Argentina. Cuadernos de Desarrollo Rural, I3(78), 97-122. https://doi.org/10.III44/Javeriana.cdr13-78.iscc

\footnotetext{
* Artículo de investigación.

** Becaria de investigación posdoctoral Conicet-Cátedra de Agroecología, Facultad de Ciencias Agrarias y Forestales, Universidad Nacional de La Plata (FCAyF-UNLP), Argentina. Correo electrónico: marilublandi@hotmail.com. Orcid: http://orcid.org/0000-0002-6995-5783

*** Coordinador del área Desarrollo Forestal Sustentable de la Dirección de Bosques, Ministerio de Ambiente y Desarrollo Sustentable de la Nación, Argentina. Correo electrónico: manhattansioux@gmail. com. Orcid: http://orcid.org/0000-0002-9348-7270

**** Docente investigadora de la cátedra de Agroecología, en la Facultad de Ciencias Agrarias y Forestales de la Universidad Nacional de La Plata. Correo electrónico: agustinagargoloff@gmail.com. Orcid: http:// orcid.org/0000-000I-6193-0090

***** Profesor titular de la cátedra de Agroecología en la Facultad de Ciencias Agrarias y Forestales de la Universidad Nacional de La Plata. Investigador de la Comisión de Investigaciones Científicas de la provincia de Buenos Aires (CIC), Argentina. Correo electrónico: sarandon@agro.unlp.edu.ar. Orcid: http:// orcid.org/0000-000I-5197-3652
} 


\title{
Resumen
}

En el presente trabajo se analizaron y compararon indicadores de prácticas de manejo que tienen efectos sobre la vegetación cultivada y no cultivada: factores que acarrean impactos en la agrobiodiversidad, en modelos de producción bajo invernadero y al aire libre, y que corresponden a agricultores de diferentes orígenes étnicos del cinturón hortícola platense. Se identificaron, mediante entrevistas desarrolladas a profundidad, conocimientos y percepciones que versan sobre la agrobiodiversidad. Se observó que los agricultores que adoptaron el sistema de producción bajo invernadero tienen manejos y visiones que tienden a disminuirla en comparación con los que cultivan al aire libre. Dentro de estos grupos, los de origen boliviano promueven una mayor agrobiodiversidad en comparación con los de origen europeo.

\section{Palabras clave:}

agroecología; agroecosistemas; horticultores; desarrollo rural; sustentabilidad

\section{Practices, Knowledge, and Perceptions that Hinder the Preservation of Agro-biodiversity. The La Plata Horticultural Belt Case, Argentina}

\begin{abstract}
This work analyzed and compared management practices indicators that have effects on cultivated and uncultivated vegetation: factors impacting agrobiodiversity on greenhouse and outdoor production models corresponding to farmers from different ethnic origins in the horticultural belt of La Plata. We identified knowledge and perceptions regarding agrobiodiversity through in-depth interviews. We also observed that the farmers who adopted the greenhouse production system have a management and vision that tends to diminish agrobiodiversity compared to those that grow their crops outdoors. Out of these groups, those of Bolivian origin promote a greater agrobiodiversity in comparison with those of European origin.
\end{abstract}

\section{Keywords:}

agroecology; agroecosystems; horticulturists; rural development; sustainability 


\section{Introducción}

En los últimos años ha comenzado a reconocerse la importancia de la agrobiodiversidad y su estrecha interrelación con la agricultura sustentable (Sarandón, 2009). La agrobiodiversidad incluye todos los componentes de la diversidad biológica que constituyen los agroecosistemas y que son relevantes para la agricultura, al igual que para la alimentación (Toledo Machado, 2007). La agrobiodiversidad puede subsidiar el funcionamiento del agroecosistema al mejorar procesos ecológicos tales como el reciclaje de nutrientes, el control biológico de plagas, y la conservación del agua y del suelo (Altieri y Nicholls, 2007); aspectos fundamentales para el diseño de sistemas agrícolas sustentables.

A pesar de que cada vez haya una mayor conciencia mundial sobre la importancia (UNEP, 2000) del asunto descrito, la agricultura que se basa en el enfoque de la revolución verde, no la tiene en cuenta, y es la principal causa de su disminución. Dicho enfoque se basa en la simplificación del sistema que lo limita a pocos cultivos de alto valor económico, y en la eliminación de ambientes semi-naturales; por tanto, los procesos ecológicos que genera la agrobiodiversidad presente en las fincas, deben ser reemplazados por insumos químicos como insecticidas, funguicidas y herbicidas, generando graves daños ecológicos y sociales (Toledo, 2005; Gliessman, 20I4).

En el análisis de la agrobiodiversidad, se ha reconocido la gran importancia de los aspectos culturales, considerándolos un componente más de la agrobiodiversidad (UNEP, 2000). Organizaciones como la Unesco y tratados internacionales como el Convenio sobre Diversidad Biológica (CDB) reconocen la importancia del conocimiento local en el manejo sustentable de los recursos naturales, entre ellos la biodiversidad (UNEP, 2000). En este sentido, se entiende que los objetivos y los conocimientos de los agricultores, al igual que la valoración que hagan de la agrobiodiversidad, determinan los niveles de dicho factor. Por ejemplo, el agricultor es quien administra la agrobiodiversidad, al decidir el diseño de los cultivos, la selección de variedades y el control de plagas y enfermedades (Stupino et al., 20I4). Esto demuestra la intima relación que se establece entre el conocimiento y las percepciones que los agricultores tienen de sus prácticas agrícolas. Por ello, Gargoloff, Abonna y Sarandón (2010) plantean la importancia de estudiar de manera conjunta las prácticas agrícolas según los conocimientos verbalizados y las percepciones de los agricultores, ya que cada una es causa, y a la vez, consecuencia de la otra (Toledo, 2005).

Además de esta estrecha relación que se teje entre los conocimientos y las prácticas, muchas veces entran en juego presiones del contexto como el asesoramiento técnico, además de diversas políticas, y el mercado, cuyas consecuencias consisten en 
que los conocimientos de los agricultores no se traduzcan finalmente en prácticas de manejo definidas (Soares Schenkel et al., 2007). En otras palabras, los saberes locales consisten en sistemas de conocimientos holísticos, acumulativos, dinámicos y abiertos, que se construyen con base en múltiples experiencias locales trans-generacionales, y que se forjan en permanente vinculo con factores del contexto. Por tanto, se organizan en constante adaptación a las dinámicas tecnológicas y socioeconómicas del territorio.

En el cinturón hortícola platense, que se encuentra situado en Buenos Aires, Argentina, durante mucho tiempo se ha cultivado una gran diversidad de especies en sistemas al aire libre con bajas inversiones de capital. Sin embargo, en las últimas décadas, los agricultores han reemplazado gran parte de los cultivos al aire libre por el cultivo bajo invernadero, que si bien aparenta ser más rentable, acarreó serios problemas ecológicos, económicos y sociales (Blandi et al., 2015). Dicha tecnología, que se asocia con un gran paquete de insumos químicos, representa la máxima simplificación de los sistemas hortícolas, pues limita el número de especies cultivadas solo a las más rentables, en cuyos casos se utilizan plaguicidas de una forma intensiva, y se eliminan ambientes seminaturales (figura I).

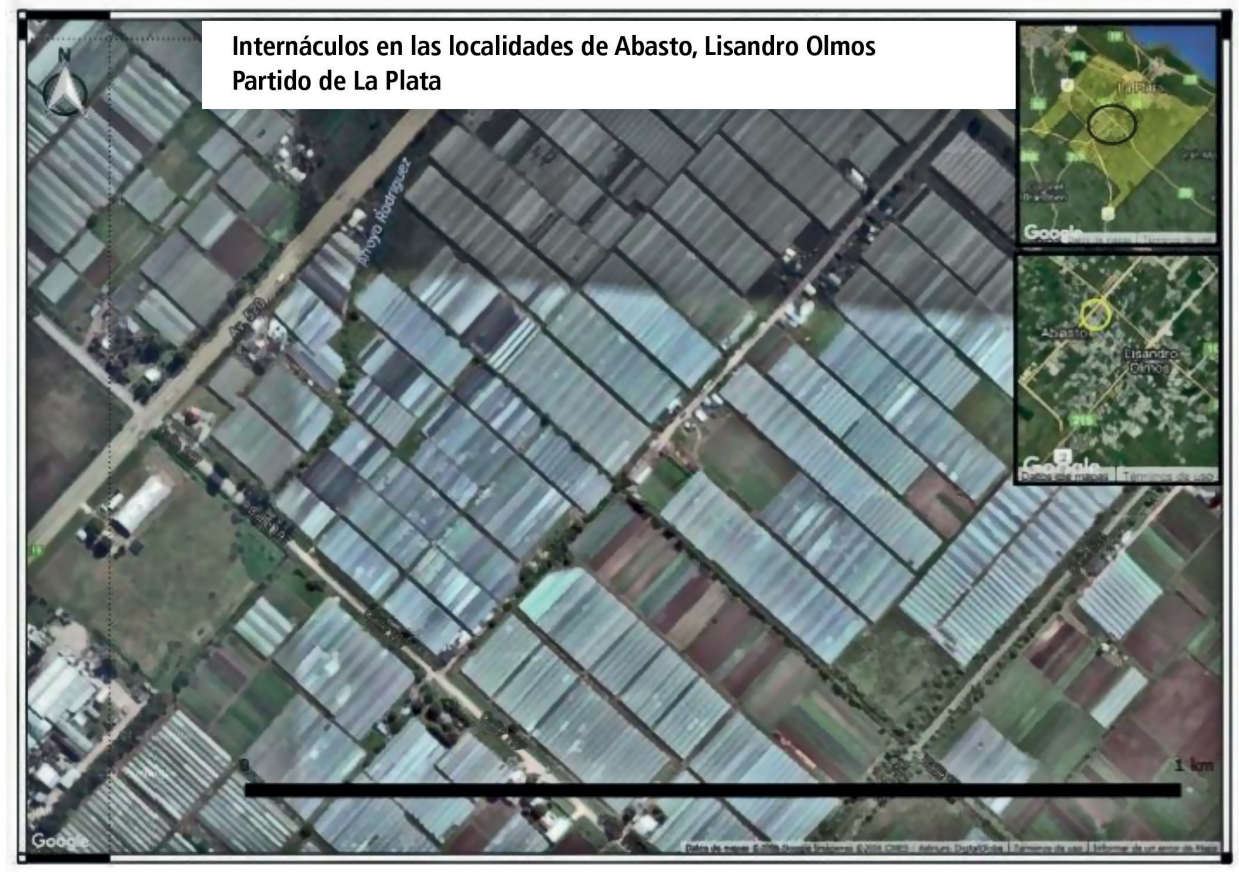

Figura 1. Imagen de invernáculos del Cinturón Hortícola Platense, Buenos Aires, Argentina, entre las localidades de Abasto y Lisandro Olmos Fuente: Google 
Según García (20I4), la incorporación del invernadero comenzó en la década de I980, cuando el cinturón hortícola estaba poblado, en su mayoría, por agricultores italianos. El perfil de los productores era bastante homogéneo: pequeños y medianos productores, con bajas inversiones de capital y con producción diversificada. Sin embargo, el invernadero empezó a incorporase al territorio en forma incipiente, donde se realizaba un manejo similar al de los cultivos al aire libre. En la década del 90 se expandió fuertemente su superficie; crecimiento liderado por agricultores de origen europeo o sus descendientes. También ocurrieron grandes migraciones de trabajadores de Bolivia al sector hortícola, en donde cumplieron papeles de peones o medieros. Durante esta década, el sector hortícola platense profundizó la incorporación del invernadero, junto con un paquete de agroquímicos - plaguicidas y fertilizantes- que transformaron a La Plata en el cinturón hortícola más tecnificado del país (García, 20I4). A partir del año 2002, fueron los agricultores de nacionalidad boliviana quienes continuaron con la incorporación del invernadero. Actualmente, el área bajo cubierta ya ha alcanzado más del $60 \%$ de la superficie del territorio (Selis, 20I2; García, 20Ira).

La diversidad cultural que hay en el territorio lleva a pensar que, tal vez, existan diferencias en las formas de cultivar de los agricultores, y que los conocimientos y las percepciones asociadas a esas prácticas también sean diferentes según sus orígenes. Según Bédard (2015), existen diferencias entre la visión occidental -o la de la sociedad moderna- y la andina. Mientras que para la primera el ser humano es el centro del universo, para la segunda el hombre se ubica en una relación de horizontalidad con respecto al resto de la naturaleza. Si bien los agricultores bolivianos aprendieron a cultivar de forma comercial en La Plata, lo que pudo conllevar una pérdida de características netamente campesinas, se podría pensar que este actor social se encuentra en una condición intermedia, con identidad porosa: "horticultor capitalista con rasgos campesinos” ya que, siendo agricultores, todavía conservan algunas características campesinas, tales como el aporte del trabajo físico, y el papel decisivo de la mano de obra familiar (García, 20ıb).

La agrobiodiversidad es un concepto complejo, cuyo abordaje no resulta sencillo (Bunnell, 2008). Por tanto, para evaluar su manejo se debe transformar este concepto complejo de acuerdo con valores claros y fáciles de interpretar; que permitan sintetizar mucha información. En ese sentido el desarrollo de indicadores, utilizados a nivel regional y de finca, puede conformar una solución (Abbona et al., 2007; Astier et al., 2011).

Se ha comprobado que el uso de esta metodología es apropiado para simplificar aspectos complejos. Sin embargo, para analizar los conocimientos y percepciones 
de los agricultores en relación con la agrobiodiversidad, se requiere además utilizar metodologías de tipo cualitativo (Minayo, 20I2; Miles, Huberman y Saldana, 2013). En este sentido, Minayo y Minayo-Gómez (2003) consideran que en la investigación es deseable la utilización complementaria de metodologías cuantitativas y cualitativas. Mientras que las del primer tipo trabajan simplificando la información con datos concretos, las del segundo profundizan en la comprensión de la complejidad de fenómenos y hechos.

En este contexto, se presupone que el cultivo bajo invernadero promueve una menor agrobiodiversidad en comparación con el cultivo que se desarrolla al aire libre. Además, acarrea disminuciones de conocimientos en relación con la conservación de la agrobiodiversidad; cambios que fomentan en los agricultores, ya que al simplificar al máximo el sistema, reemplazando sus funciones por insumos químicos, no sería necesario manejar los procesos ecológicos. Es así como se asumen las siguientes hipótesis: los agricultores que cultivan bajo invernadero cuentan con prácticas, conocimientos y percepciones menos preservadoras de la agrobiodiversidad que los agricultores que cultivan al aire libre. A su vez, los agricultores de origen europeo tienen conocimientos y percepciones menos preservadoras de la agrobiodiversidad que aquellos de origen boliviano.

Así pues, el objetivo del trabajo fue analizar y comparar prácticas de manejo, conocimientos y percepciones que versan sobre la agrobiodiversidad en grupos de agricultores de diferentes orígenes que cultivan bajo invernadero y al aire libre, y que se sitúan en el cinturón hortícola platense.

\section{Metodología}

El trabajo se realizó en el cinturón hortícola platense, provincia de Buenos Aires, Argentina. Se utilizó la metodología de estudio de casos (Yin, 20I3). Cuando la pregunta de investigación se orienta a conocer cómo o por qué ocurren determinados eventos, el estudio de casos es la estrategia de investigación más apropiada (Yin, 2013). Según el mismo autor, el estudio de casos se prefiere cuando se examinan eventos contemporáneos y no se trata de controlarlos, sino de describir y comprender su funcionamiento. Tal metodología privilegia el análisis de los casos seleccionados en forma integral. Esto es necesario debido a la complejidad de la realidad hortícola y a la temática abordada. En el estudio de caso se selecciona una muestra teórica, es decir: se seleccionan casos que probablemente pueden comprobar la teoría (Eisenhardt, 1989). 
Así, según Yin (2013), los resultados del estudio de un caso pueden generalizarse y extenderse a otros que representan condiciones teóricas similares. Para conformar la muestra, los agricultores satisficieron los criterios de selección establecidos, que en este trabajo fueron el grado de tecnificación y el origen del productor. Respecto al número de casos, estos deben adicionarse hasta la saturación de la teoría (Eisenhardt, 1989). Eisenhardt (199I) sugiere que el número de casos apropiado depende del conocimiento sobre el tema del que se disponga, y de la información que se pueda obtener por medio de la incorporación de estudios de casos adicionales. Eisenhardt (1989) sugiere entre cuatro y diez casos, y afirma que con ese rango se trabaja bien, siempre y cuando no haya un número ideal. En el presente trabajo, se entrevistaron agricultores hasta que las entrevistas no aportaron nueva información (Eisenhardt, 1989), resultando un total de 16 agricultores.

Para la elección de los agricultores, se tuvo en cuenta la clasificación de Hang et al. (2010), puesto que caracteriza los sistemas de producción que constituyen el cinturón hortícola platense. Así, se seleccionaron grupos de agricultores representativos, que se describen a continuación:

\section{Grupo i. Agricultores de origen europeo que cultivan bajo invernadero (AEI)}

Agricultores de origen europeo -o descendientes de ellos-, con fincas con producción preponderantemente bajo invernadero, que oscila entre tres y 12 hectáreas, que realizan entre uno y cuatro cultivos. Tales agricultores son propietarios de la tierra, en la que predomina la mano de obra asalariada y el alto grado de capitalización. Dichos agricultores comercializan sus productos en mercados concentradores y supermercados.

\section{Grupo 2. Agricultores de origen boliviano que cultivan bajo invernadero (ABI)}

Agricultores de nacionalidad boliviana que poseen fincas con producción en su mayoría bajo invernaderos. Los terrenos varian entre una y cuatro hectáreas, en donde realizan entre seis y ro cultivos. Los agricultores no son propietarios de la tierra, predomina el trabajo familiar y comercializan predominantemente a culata de camión, es decir: con base en una modalidad de venta en la que el intermediario compra las verduras directamente en la finca, pactando cantidad y precio en el momento de la transacción. 


\section{Grupo 3. Agricultores de origen europeo que cultivan al aire libre (AEAL)}

Agricultores de origen europeo, o descendientes de ellos: poseen fincas con la mayor parte de su producción al aire libre, y que oscilan entre tres cuartos de hectárea y 13 hectáreas. En dichos terrenos realizan entre ro y 19 cultivos. Dichos agricultores son propietarios de la tierra, en donde predomina el trabajo familiar que involucra algunos trabajadores externos temporales que comercializan la mayor parte de sus productos en mercados concentradores.

\section{Grupo 4. Agricultores de origen boliviano que cultivan al aire libre} (ABAL)

Agricultores de nacionalidad boliviana que cuentan con fincas de producción al aire libre cuyos terrenos oscilan entre dos y ir hectáreas. En ellas realizan entre Io y 6 cultivos, pero no son propietarios de la tierra. Además predomina el trabajo familiar, que envuelve escasa incorporación de tecnología. Los agricultores además comercializan la mayor parte de su mercadería en la finca directamente con el consumidor, y lo que resta lo envían a mercados concentradores.

A lo largo de los años 2012 y 2013 se realizaron, en promedio, cuatro entrevistas a cada uno de los agricultores en sus fincas, en diferentes momentos del año. En las primeras dos visitas se realizaron entrevistas de tipo semiestructurado, con el objetivo de relevar información acerca de las prácticas agrícolas y analizar cómo estas impactan la agrobiodiversidad. Para tal propósito se empleó una metodología de indicadores y, luego, en las últimas dos, se realizaron entrevistas que permitieron analizar a profundidad las temáticas descritas, y comparar múltiples conocimientos y percepciones de los diferentes grupos de agricultores. Las entrevistas fueron transcritas en su totalidad y sistematizadas. El análisis cualitativo se basó en el material discursivo, que fue ordenado en categorías.

\section{Metodología de indicadores}

Se utilizó la metodología de indicadores para evaluar el manejo de la agrobiodiversidad (Nahed, 2008; Bunnell, 2008). Por un lado, se consideró la agrobiodiversidad "planificada o intencional", que comprende cultivos y animales 
incluidos en el agroecosistema por decisión del agricultor, y que varía en forma directa de acuerdo con el manejo que se da a los cultivos. Por otra parte, es de gran importancia la agrobiodiversidad "asociada o no planificada”, que es representada por la flora y la fauna del suelo, los herbívoros, las plagas, los descomponedores y depredadores benéficos, los cercos vivos y las zanjas, así como las márgenes de las fincas. Estos son influenciados de manera indirecta por el tipo de manejo que se $\mathrm{da}$ a los terrenos (Flores, 20I2). Tales conceptos dieron origen a dos descriptores fundamentales para analizar el efecto que las prácticas de manejo tienen sobre la agrobiodiversidad: uno que corresponde a la cuantificación de la diversidad cultivada, y otro a la evaluación de la diversidad no cultivada. Para cada uno de estos descriptores se desarrolló un conjunto de indicadores.

\section{I.I Construcción de indicadores}

Para la investigación, se empleó la metodología y el marco conceptual propuesto por Sarandón et al. (2014), y se siguieron además los lineamientos de Acevedo-Osorio y Angarita-Leiton (20I3), y Astier et al. (20II). Como base, en el estudio descrito se utilizó el conjunto de indicadores desarrollados por Flores (20I2), y se trabajó sobre ellos para adaptarlos y modificarlos de acuerdo con los fines de este estudio.

En ese contexto se entiende por indicador a una variable, seleccionada y cuantificada que hace clara una tendencia que de otra forma no sería fácilmente detectable. Se eligieron indicadores fáciles de obtener e interpretar; medidores que brindan la información necesaria para los estudios y que permiten detectar tendencias representativas en el ámbito de unidad productiva, que corresponde en este caso a la finca. Los descriptores son indicadores “robustos”; es decir, que están conformados a su vez por otros indicadores (tabla I).

En ese contexto, con el fin de permitir la comparación de las fincas y facilitar el análisis, los indicadores se estandarizaron mediante su transformación a una escala de o a i, siendo i el mayor valor de conservación de la agrobiodiversidad y o el más bajo. Por otra parte, para el análisis de los resultados y la detección de los puntos críticos, se estableció previamente un valor umbral de 0.5 para ambos descriptores. En tal punto se consideró que en cualquier descriptor que se situara por debajo de tal valor el manejo del agroecosistema no cumpliría con los requisitos de conservación de la agrobiodiversidad que eran idóneos. Los resultados se representaron gráficamente mediante el diagrama tipo tela de araña. 
TABla 1. Indicadores ecológicos de agrobiodiversidad empleados en fincas de producción hortícola al aire libre y bajo invernáculo, del Cinturón Hortícola Platense en Buenos Aires, Argentina

\begin{tabular}{|c|c|c|}
\hline DESCRIPTOR & INDICADOR & ESTANDARIZACIÓN \\
\hline \multirow[t]{4}{*}{$\begin{array}{l}\text { I. Diversidad } \\
\text { cultivada }\end{array}$} & $\begin{array}{l}\text { I.I. Número } \\
\text { de especies } \\
\text { cultivadas por } \\
\text { hectárea }\end{array}$ & $\begin{array}{l}\text { (I) Entre dos o más especies cultivadas por hectárea; (0.75) } \\
\text { entre I.5 y menos de dos especies cultivadas por hectárea; } \\
\text { (0.50) entre una y I.5 especies cultivadas por hectárea; (0.25) } \\
\text { entre } 0.5 \text { y menos de una especies cultivada por hectárea; } \\
\text { (0) menos de } 0.5 \text { especies cultivadas por hectárea. }\end{array}$ \\
\hline & $\begin{array}{l}\text { I.2. Diversidad } \\
\text { espacial }\end{array}$ & $\begin{array}{l}\text { (I) Cultiva en franjas, líneas intercaladas, al azar y utiliza } \\
\text { cultivos de cobertura y cercos vivos; ( } 0.75) \text { realiza tres de las } \\
\text { opciones mencionadas; ( } 0.50 \text { ) realiza dos de las opciones } \\
\text { mencionadas; ( } 0.25 \text { ) realiza una de las opciones mencionadas; } \\
\text { (o) no realiza ninguna de las opciones mencionadas arriba. }\end{array}$ \\
\hline & I.3. Rotaciones & $\begin{array}{l}\text { (I) Rotaciones planificadas que incorporan } \\
\text { especies de diferentes familias; ( } 0.75) \text { rotaciones } \\
\text { planificadas de especies de igual familia, } \\
\text { ( } 0.50) \text { rotaciones no planificadas pero que incorporan } \\
\text { especies de diferentes familias; ( } 0.25) \text { rotaciones no } \\
\text { planificadas de especies de igual familia, o rotaciones } \\
\text { dentro del año; (o) rotaciones inexistentes. }\end{array}$ \\
\hline & $\begin{array}{l}\text { I.4. Reproducción } \\
\text { de semillas }\end{array}$ & $\begin{array}{l}\text { (I) Reproduce el roo \% de las semillas que utiliza; ( } 0.75 \text { ) } \\
\text { reproduce el } 75 \% \text { de las semillas que utiliza; (0.50) reproduce el } \\
50 \% \text { de las semillas que utiliza; ( } 0.25 \text { ) reproduce el } 25 \% \text { de las } \\
\text { semillas que utiliza; ( }) \text { no reproduce las semillas que utiliza. }\end{array}$ \\
\hline $\begin{array}{l}\text { 2. Diversidad } \\
\text { natural }\end{array}$ & $\begin{array}{l}\text { 2.I. Distribución } \\
\text { y relación de las } \\
\text { áreas cultivadas } \\
\text { y seminatural }\end{array}$ & $\begin{array}{l}\text { (I) El área cultivada representa menos del } 70 \% \text { de la superficie, } \\
\text { y el resto se encuentra en forma de bordes, franjas y corredores } \\
\text { planificados por el agricultor; ( } 0.75 \text { ) el área cultivada representa } \\
\text { entre el } 80 \text { y el } 89 \% \text { de la superficie y el resto está planificada } \\
\text { en forma de bordes, franjas y corredores; ( } 0.50 \text { ) el área cultivada } \\
\text { representa más del } 70 \% \text { de la superficie y el resto de la } \\
\text { superficie se encuentra sin planificar; ( } 0.25 \text { ) el área cultivada } \\
\text { representa entre el } 80 \text { y } 89 \% \text { de la superficie, y el resto del área } \\
\text { no está planificada; ( } 0 \text { ) el área cultivada representa más del } 90 \\
\% \text { de la superficie y el resto del área se encuentra sin planificar. }\end{array}$ \\
\hline & $\begin{array}{l}\text { 2.2. Uso de } \\
\text { pesticidas }\end{array}$ & $\begin{array}{l}\text { (I) No utiliza pesticidas o utiliza solo productos biológicos } \\
\text { o naturales; ( } 0.75 \text { ) productos exclusivamente de categorías } \\
\text { poco tóxicas y con baja frecuencia; ( }(0.50) \text { productos de } \\
\text { todas las categorías toxicológicas en baja frecuencia (en } \\
\text { promedio, dos aplicaciones por mes); ( } 0.25) \text { productos, en } \\
\text { mayor proporción, de las categorías más toxicas en alta } \\
\text { frecuencia (en promedio, ocho aplicaciones por mes); (o) } \\
\text { productos exclusivamente de las categorías más toxicas en } \\
\text { alta frecuencia (en promedio, ocho aplicaciones por mes). }\end{array}$ \\
\hline
\end{tabular}

FuENTE: elaboración propia con base en el trabajo de Flores (20I2) 


\section{I.2 Entrevista}

Con base en los indicadores definidos, se construyó una entrevista semiestructurada (Ander-Egg, 2000; Marradi, Archentti y Piovani, 2007) y se entrevistó a los agricultores seleccionados. Además se realizaron observaciones y se utilizó un diario de campo (Taylor Bogdan y DeVault, 2015). Yin (2013) recomienda la utilización de múltiples fuentes de datos, y el cumplimiento del principio de triangulación, que es la combinación de diferentes técnicas que permiten analizar las convergencias y divergencias de la información que fue obtenida mediante diferentes fuentes. En el caso del presente estudio, se trianguló la información que fue relevada con las entrevistas, con la observada en campo, para garantizar la validez interna de la investigación. En ese contexto, la presencia de fauna, malezas y vegetación alrededor del lote se determinó principalmente por observaciones a campo.

\section{Análisis cualitativo}

Con el fin de analizar y comparar múltiples conocimientos y percepciones que corresponden a los diferentes grupos de agricultores, se utilizó la entrevista en profundidad (Ander-Egg, 2000); procedimiento implementado luego de la entrevista semiestructurada. Para utilizar esta técnica, se realizó un guión de entrevista que incluía los temas a tratar por el entrevistador. En tal contexto, no hubo preguntas cerradas o con opciones de respuesta para elegir.

Luego, las respuestas fueron transcriptas en su totalidad. En adelante se siguieron los lineamientos de Minayo (20I2) y Miles et al. (20I3) para su análisis. En ese contexto se realizaron varias lecturas de las transcripciones, con el objetivo de delimitar fragmentos textuales según las categorías descriptas a continuación:

\section{Manejo de los cultivos:}

* Número de especies cultivadas: se profundizó en la importancia y en la justificación de la cantidad de especies que cada agricultor cultiva.

* Rotaciones: se indagó sobre la importancia y los conocimientos alusivos a esta práctica.

* Reproducción de semillas: se preguntó sobre la importancia y posibilidad de realizar esta práctica.

* Presencia de fauna: se indagó sobre su importancia y las distintas percepciones que los agricultores tenían sobre el tema. 
* Presencia de malezas: se profundizó sobre su importancia y las percepciones de los agricultores.

* Presencia de vegetación alrededor del lote: se indagó sobre su importancia.

* Utilización de plaguicidas: se analizó según la clasificación de la Organización Mundial de la Salud (OMS). Se organizó la información de acuerdo con las siguiente catalogación: Categoría I. Sumamente peligroso o muy peligroso; Categoría II. Moderadamente peligroso; Categoría III. Poco peligroso; Categoría IV. Normalmente no ofrece peligro.

Los fragmentos fueron agrupados de forma manual en su categoría correspondiente. Luego, el análisis y la interpretación se centraron en el material discursivo acumulado en cada categoría, y se organizaron de una forma argumental y narrativa (Minayo, 2012; Miles et al., 2013).

\section{Resultados}

\section{I. Indicadores de manejo de agrobiodiversidad}

Los agricultores que cultivan en invernaderos, independientemente de sus nacionalidades, realizan un manejo de sus fincas que disminuye la agrobiodiversidad (figura 2A). En lo que compete a la agrobiodiversidad cultivada, cuentan con una escasa diversidad espacial; no realizan un adecuado esquema de rotaciones, y no reproducen sus propias semillas. Además, a los agricultores de origen europeo se les suma como factor crítico el bajo número de cultivos realizados. En cuanto a la conservación de la agrobiodiversidad no cultivada se observa que en ambos grupos de agricultores los principales factores críticos fueron la escasa o nula relación de área cultivada con área seminatural, y el uso indiscriminado de plaguicidas de gran toxicidad. Como aporte a la conservación de la agrobiodiversidad se puede destacar en los agricultores de origen boliviano un alto número de especies cultivadas (figura $2 \mathrm{~A}$ ).

Por el contario, los agricultores que cultivan al aire libre realizan varias prácticas que favorecen la agrobiodiversidad sin importar el origen del agricultor (figura $2 \mathrm{~B}$ ). Con respecto a la agrobiodiversidad cultivada se destaca, por una parte, el esquema planificado de rotaciones que caracteriza el grupo y, por otra, el alto número de 
especies cultivadas. Con relación a la agrobiodiversidad no cultivada se destaca el bajo uso de plaguicidas, menor que el que se da bajo invernadero. Asimismo, en los agricultores de origen boliviano la reproducción de la propia semilla y la adecuada distribución y relación de áreas cultivadas y seminaturales se identificaron también como aportes a una buena diversidad espacial (figura $2 \mathrm{~B}$ ).

A. Invernáculo

B. Aire libre
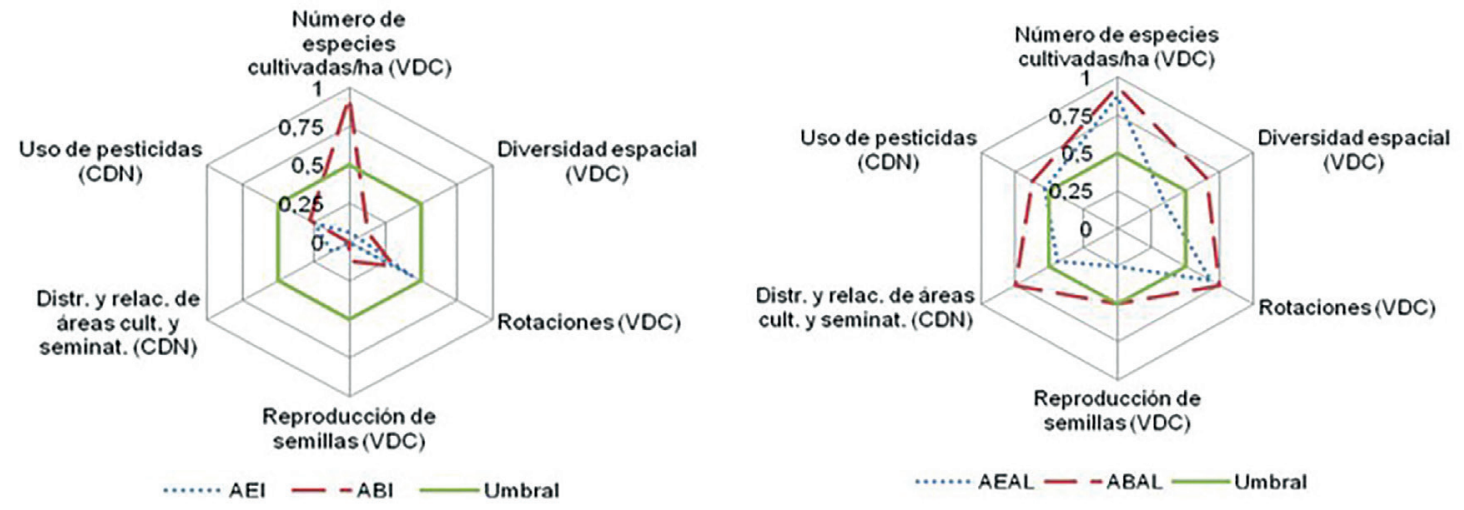

Figura 2.

A. Diagrama en tela de araña que representa los indicadores de agrobiodiversidad de fincas de producción hortícola bajo invernáculo. B. Diagrama en tela de araña que representa los indicadores de agrobiodiversidad de fincas con producción hortícola al aire libre. En línea punteada se muestran los agricultores de origen europeo y en líneas de trazos los agricultores de origen boliviano que se sitúan en el Cinturón Hortícola Platense, en Buenos Aires, Argentina. En ambos casos la línea continua representa el valor umbral.

Referencias: AEI. Agricultor de origen europeo con invernáculo; ABI. Agricultor de origen boliviano con invernáculo; AEAL. Agricultor de origen europeo al aire libre; ABAL. Agricultor de origen boliviano al aire libre.

FuENTE: elaboración propia 


\section{Conocimientos y percepciones de los diferentes grupos de agricultores en relación con la agrobiodiversidad}

\section{Número de especies estudiadas:}

Los agricultores de origen europeo que cultivan bajo invernadero producen pocas especies por una cuestión organizativa, ya que para ellos es más conveniente que sus trabajadores se especialicen en pocos cultivos. Los de origen boliviano que cultivan bajo invernadero estuvieron de acuerdo con esa opinión. Por su parte, los agricultores de origen europeo que cultivan al aire libre destacaron la importancia de cultivar diversas especies, ya que de esta manera solo algunos cultivos serían afectados por la presencia de plagas. Dichos individuos agregaron que al mercado siempre hay que llevar varios artículos, pues algunos valen más que otros dependiendo de la época del año. En tanto, los agricultores de origen boliviano que cultivan al aire libre también señalaron la importancia de cultivar diferentes especies y, en general, afirmaron que es más importante producir diversidad que cantidad por dos motivos: el primero es que si hay problemas de plagas, solo algunos cultivos serían afectados, y el segundo, que sus clientes buscan diversidad de productos. Además, un agricultor comentó que buscaba sembrar en un mismo surco dos tipos de verdura para así ahorrar trabajo en relación a la preparación del terreno y aprovechar el espacio que hay entre cada surco.

Todas las verduras no son, hay unas verduras que son más lentas y otras son más rápidas... utilizo el mismo surco para hacer dos clases de verduras... aprovechás más la tierra, porque estás utilizando el doble el surco, y el abono también... (agricultor de origen boliviano que cultiva al aire libre) (Comunicación personal)

Otro agricultor explicó que el cultivo de diversas especies altas puede hacer que no crezca el cebollín (Cyperus rotundus), maleza con la que los agricultores tienen mayores problemas.

\section{Rotación de cultivos:}

Los agricultores de origen europeo que cultivan bajo invernadero reconocieron que la rotación es importante, pero afirmaron que por medio del manejo intensivo que realizan, cultivando tomate (Solanum lycopersicum) sobre tomate, no tienen tiempo para incluirla. 
Rotar es muy importante, porque qué pasa, nosotros estamos haciendo siempre tomate sobre tomate, llega un momento que no sé si el suelo va a responder, sería bueno rotar tomate, un poco de hoja viste, o pimiento (Capsicum annuum), eso es muy bueno, pero nosotros como hacemos exclusivamente tomate, no nos dan los tiempos... (Comunicación personal de agricultor de origen europeo que cultiva en invernadero)

Otro agricultor aclaró que su forma de producir funciona, y hasta que no haya señales de disminución de rendimiento continuará con el mismo procedimiento. Los agricultores de origen boliviano que cultivan bajo invernadero reconocieron la importancia de las rotaciones dentro del año, es decir: de plantar alguna verdura de hoja para “sacar el exceso de nutrientes” después de cosechar el tomate.

Los agricultores que cultivan al aire libre, independientemente de su origen, reconocen la importancia de esta práctica porque favorece la interrupción de los ciclos reproductivos de los insectos plagas y por la extracción de diferentes nutrientes.

...sé que no se puede poner siempre lo mismo porque, yo sé que lo mismo siempre extrae, porque todas las plantas extraen diferentes nutrientes, entonces si yo ...voy a poner siempre en el mismo lote se van a agotar (los nutrientes), rápido, entonces... (Comunicación personal provista por agricultor de origen europeo que cultiva al aire libre)

Reproducción de semillas:

Los agricultores de origen europeo con invernaderos advierten que las empresas encargadas de comercializar las semillas eligen las variedades a utilizar todos los años, y que la única opción que tienen es comprarlas. Comentaron que antes se hacían, pero que demandaban mucho trabajo, de modo que los tiempos de producción no alcanzan para realizar todas las tareas. Los agricultores de origen boliviano que cultivan en invernadero indicaron que no las pueden reproducir porque la mayoría de ellas son híbridas y con el tiempo van perdiendo calidad. Por otra parte, los agricultores de origen europeo que trabajan al aire libre reconocen que la anteriormente mencionada es una buena estrategia porque las semillas cada vez son más caras en las casas de insumos. Por su lado, los agricultores de origen boliviano al aire libre destacaron la importancia de producir sus propias semillas, ya que las compradas son caras. Además, porque se intercambian en las ferias de semillas. 


\section{Presencia de fauna:}

Los agricultores de origen europeo con invernaderos aclararon que algunos insectos podrían tener algún grado de importancia, pero es imposible que queden vivos debido a la cantidad de plaguicidas que utilizan. En ese contexto, los agricultores de origen boliviano con invernaderos reconocieron la importancia de las abejas (Apis mellifera). Un agricultor afirmó que cuando una abeja entra al invernadero se muere, aunque no se fumigue, ya que se golpea mucho contra el nylon. Sin embargo, otro sostuvo que hay abejas dentro de su invernadero. Los agricultores también reconocieron la función de otro insecto, la "Vaquita de San Antonio" (Diabrotica speciosa), que combate los pulgones (Fam. Aphididae). Sin embargo, ya que los insectos en su mayoría son dañinos, se aplican plaguicidas y todos terminan muriendo. Los agricultores de origen europeo que cultivan al aire libre reconocieron la importancia de algunos insectos, como las abejas, dada su función de polinización. Los agricultores de origen boliviano al aire libre también hicieron referencia a las lombrices (Fam. Lumbricidae) puesto que oxigenan la tierra.

\section{Presencia de malezas:}

Los agricultores de origen europeo con invernadero aclararon que el terreno tiene que estar libre de malezas, tanto dentro como fuera del invernadero, porque si persisten atraen insectos plaga. Los agricultores de origen boliviano con invernaderos nombraron a la gramilla (Cynodon dactylon) por beneficiar la tierra, pero aclararon que la mayoría de las malezas toman los nutrientes del suelo y no los devuelven, lo cual disminuye el rendimiento de los cultivos. Por su parte, los agricultores de origen europeo al aire libre afirmaron que estas son enemigas dentro del cultivo, y que se las intenta combatir con el uso de herbicidas o eliminándolas de manera mecánica. Sin embargo, reconocieron que siempre están presentes y que habitualmente se termina cosechando junto a ellas.

\section{Presencia de vegetación alrededor del lote:}

Los agricultores que cultivan bajo invernadero eliminan totalmente la vegetación que rodea el lote. En tanto, los agricultores que desarrollan sus labores al aire libre en general la mantienen presentes, ya que no les causan grandes molestias. En ese contexto, algunos de los agricultores de origen boliviano la utilizan como alimento para caballos o como cortina para proteger sus cultivos del viento. Tales agricultores también comentaron que a esos pastos van abejas, principalmente cuando hay flores. 


\section{Utilización de plaguicidas:}

Los agricultores de origen europeo que cultivan bajo invernaderos nombraron 22 plaguicidas diferentes, ayudados por los cuadernos que utilizan los ingenieros agrónomos que los asesoran (figura $3 \mathrm{~A}$ ). Por su parte, los agricultores de origen boliviano nombraron ig (figura $3 \mathrm{~B}$ ). Ambos grupos aclararon que los plaguicidas se aplican en todos los cultivos. La frecuencia de la aplicación varía según el cultivo y el estado de los cultivos, pero, en promedio, se realizan dos aplicaciones semanales. Algunos agricultores que cultivan bajo invernadero, principalmente los de origen europeo, se muestran muy confiados en relación a su manejo fitosanitario, ya que son asesorados por ingenieros. Los agricultores bolivianos manifestaron que están obligados a manejarse como lo hacen todos los agricultores de la zona, porque de lo contario su producto podría perder calidad.

Estás obligado a echar, no es que uno lo echa porque lo quiere echar... lamentablemente estás obligado a manejarte como lo hacen todos, sino tu producto va a ser el más feo de toda la zona... y el gasto ya lo hiciste, así que... (Comunicación personal provista por agricultor de origen boliviano que cultiva bajo invernadero)

Sistema de producción bajo invernáculo
A. Agricultores de origen europeo
B. Agricultores de origen boliviano
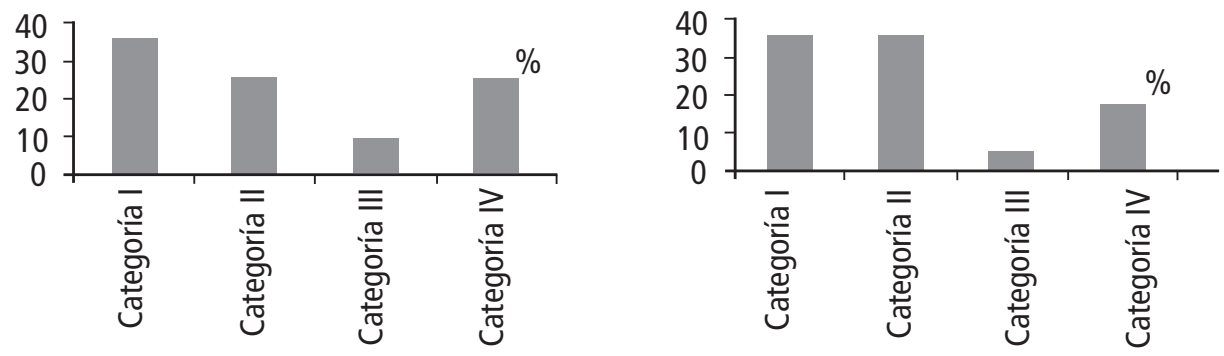

Figura 3. Categoría toxicológica de pesticidas utilizados en sistemas de producción bajo invernáculo del Cinturón Hortícola Platense en Buenos Aires, Argentina Fuente: elaboración propia 
Los agricultores de origen europeo al aire libre nombraron 20 plaguicidas diferentes (figura $4 \mathrm{~A}$ ). Con respecto a la frecuencia de utilización, ambos coincidieron en que esta depende del cultivo y de la necesidad, pero que las aplicaciones eran realizadas en promedio una vez cada is días. En ese contexto, los agricultores de origen boliviano nombraron ocho plaguicidas diferentes (figura ${ }_{4} \mathrm{~B}$ ). Con respecto a la frecuencia de utilización, dicho grupo coincidió con el grupo anterior en cuanto a que esta depende del cultivo y de la necesidad pero que, en promedio, las aplicaciones son realizadas una vez cada is días.

Sistema de producción al aire libre
A. Agricultores de origen europeo
B. Agricultores de origen boliviano
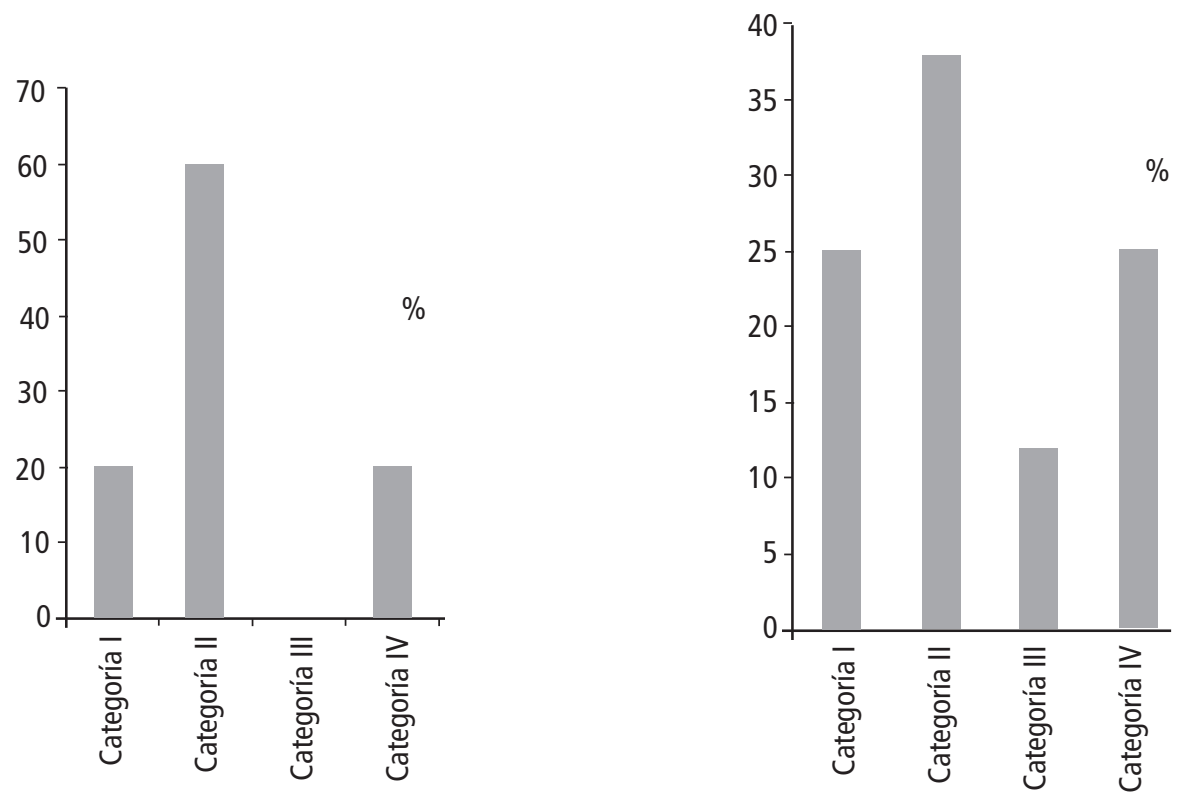

Figura 4. Categoría toxicológica de pesticidas utilizados en sistemas de producción al aire libre del Cinturón Hortícola Platense en Buenos Aires, Argentina Fuente: elaboración propia

\section{Discusión}

Los datos del presente trabajo demuestran que el modelo de producción bajo invernadero promueve una menor agrobiodiversidad que el sistema de cultivo al aire libre sin que importe el origen del agricultor. Según Nicholls (2006) lo anterior aumenta su susceptibilidad a enfermedades, plagas y variaciones climáticas, y los convierte en sistemas más dependientes de insumos externos. En contraste, la mayor 
cantidad de especies cultivadas que se encontró en los cultivos al aire libre aumenta la diversidad genética y favorece la agrobiodiversidad (Nicholls, 2006).

Otra diferencia notable que se sitúa entre los modelos de producción es que el cultivo bajo invernadero no involucra un esquema planificado de rotaciones, mientras que el cultivo al aire libre sí. Las rotaciones establecen secuencias temporales en las que se obtienen aportes de nutrientes al rotarse diferentes cultivos, o se regulan los insectos, malezas y enfermedades en tanto que los cultivos rompen con sus ciclos de vida (Nicholls y Altieri, 20I2). El agregado de los residuos de diferentes plantas al suelo ayuda a mantener la diversidad biológica, ya que cada tipo de residuo varía química y biológicamente, estimulando y/o inhibiendo diferentes organismos del suelo (Gliessman, 20I4). Por tanto, esta práctica resulta esencial para favorecer la agrobiodiversidad.

Los agricultores de origen boliviano que cultivan al aire libre reproducen muchas de las semillas que utilizan. Según Bonicatto, Marasas, Pochettino y Sarandón (2014) esta es una práctica fundamental, ya que junto a la conservación de semillas hay tradiciones y saberes asociados que solo pasan de generación en generación vía oral. Por tanto, si esta práctica no continuase, como ocurre con el cultivo bajo invernadero, se pondrían en riesgo muchos saberes que se relacionan con la producción y la conservación de especies. Actualmente, en el cinturón hortícola platense existen todavía varias especies junto al conocimiento de cómo cultivarlas, gracias a la conservación y reproducción de semillas que llevan a cabo los agricultores, ya que comercialmente han dejado de ser utilizadas desde hace tiempo (Cababié, Bonicatto y Abbona, 2014).

La utilización de plaguicidas fue otra diferencia que había entre las fincas analizadas. Los datos del trabajo confirman que el modelo de producción bajo invernadero es químico dependiente. En ese sentido, un alto uso de plaguicidas reducirá la complejidad biológica del suelo, principalmente en el grado en que estos sean de alta toxicidad. Además, los utilizados en el control de plagas son capaces de producir contaminación en suelos y aguas tanto superficiales como subterráneas, lo que envuelve un riesgo importante de intoxicación en seres vivos, incluyendo al hombre (Peres et al., 2007). Es así como con el cultivo bajo invernadero todos estos riesgos se intensifican en comparación con lo que se observa en el cultivo al aire libre.

En el sistema de cultivo al aire libre se encontraron algunas prácticas que disminuyen la agrobiodiversidad. Sin embargo, con la utilización del potencial de recursos naturales y en algunos casos con la propuesta de un rediseño del sistema (Guzman Casado y Alonso Mielgo, 2007), se requerirían menores esfuerzos para la preservación de la 
agrobiodiversidad, en comparación con el cultivo bajo invernadero. Por ejemplo, al aire libre los sistemas cuentan con vegetación seminatural en los bordes de los cultivos, en lotes de descanso y montes, y hasta pueden contar con cuerpos de agua; ambientes en donde se encuentra una gran diversidad de especies vegetales y animales, capaces de regular varios procesos ecológicos (Kaiser-Bunbury et al., in press).

Los resultados muestran que a pesar de saber que ciertas prácticas no son beneficiosas para el medio ambiente, y en consecuencia para la agrobiodiversidad, los agricultores que cultivan bajo invernadero creen que no hay otra manera de producir. En este grupo de agricultores se percibe una visión en la que el ser humano puede intervenir sobre la naturaleza casi sin límites. Esto puede deberse a que este tipo de agricultor ha incorporado avances tecnológicos, como plaguicidas, fertilizantes sintéticos, invernaderos, entre otros, que han reemplazado las funciones de la agrobiodiversidad espontánea vegetal y animal, restándoles importancia a los servicios ecológicos que eran prestados por estos (Garrido-Fernandez, 2006). Además, dichos agricultores conocen algunas prácticas que envuelven la conservación de la agrobiodiversidad, pero aseguran que estas no se pueden aplicar en producciones a gran escala. Los resultados descritos ponen en evidencia que los agricultores conocen algunas prácticas, pero, por diversos motivos, creen que no se deben implementar. Esto demuestra que existe un saber local que se va reinterpretando por el contexto ecológico y por las dinámicas culturales y económicas del territorio. En consecuencia, se debería trabajar - por medio del diálogo de saberes entre investigadores, extensionistas y agricultores- en las limitantes de su traducción, que dirigen hacia prácticas adecuadas que conserven la agrobiodiversidad. Estos datos concuerdan con Blesh y Barret (2006), quienes afirman que varias técnicas ambientalmente adecuadas fueron conocidas por agricultores tecnificados de Estados Unidos pero que no fueron consideradas posibles.

En general, si bien los agricultores que cultivan al aire libre han incorporado “innovaciones tecnológicas" como los agroquímicos y semillas hibridas, entre otros, reconocen la importancia de prácticas ecológicamente adecuadas. Cultivar una gran variedad de especies, así como rotar y no eliminar toda la vegetación espontánea, son actividades fundamentales para preservar la agrobiodiversidad de los sistemas (Gliessman, 2014). Estos datos coinciden con los encontrados por Pérez (2010), quien afirma que agricultores familiares del Cinturón Hortícola de La Plata y Gran Buenos Aires Sur, cuentan con un gran entendimiento de sus quintas y, en líneas generales, reconocen los beneficios ecológicos de la agrobiodiversidad. 
La modernización agrícola fue, y es, fuertemente estimulada en el Cinturón Hortícola Platense por medio de los mercados de insumos y concentradores de hortalizas. Estos ejercen gran presión para que los agricultores logren obtener un producto de acuerdo con determinados "estándares de calidad" que son, principalmente, el color y el tamaño homogéneos, al igual que la ausencia de marca o mancha de ningún tipo, de modo tal que para obtener ese tipo de producto se debe utilizar una gran cantidad de agroquímicos; actividad compatible con la producción bajo invernáculo (Marasas, Blandi, Dubrovsky Berensztein y Fernandez, 20I4).

Además, este cambio contó con apoyo científico y político. Esto se debe a que tradicionalmente los -agrónomos o- profesionales de las Ciencias Agrarias han sido formados de acuerdo con un modelo agrícola productivista que se basa en una intensa mecanización agrícola; en un uso creciente de agroquímicos, y en variedades mejoradas de cultivos. Por tanto, no es sorprendente que reproduzcan, en la investigación y extensión, el mismo estilo de agricultura con el que fueron formados. Desde la dimensión política, durante la década del 90, el Estado prácticamente no tuvo injerencia sobre el territorio, de modo que dejó que el sector privado se ocupara del "desarrollo" de la región. En consecuencia, es de esperar que muchos agricultores reproduzcan el discurso de la tan fomentada agricultura industrial (Blandi, 20I6; Sabogal Aguilar y Hurtado, 2008) y que crean que ya no se puedan realizar prácticas agrícolas 'atrasadas' porque no son adecuadas para los sistemas modernos de producción.

Los problemas citados fueron más evidentes en los agricultores de origen europeo que en los de origen boliviano. Se podría pensar que esas diferencias se deben al propio origen de los agricultores bolivianos, muchos de ellos con valores y creencias campesinas que actúan como freno de las netamente capitalistas. En ese sentido, varios trabajos han demostrado que los agricultores campesinos cuentan con una visión que integra al ser humano con el resto de la naturaleza, y que no lo posiciona contra ella (Toledo y Barrera-Bassols, 2008). Al mismo tiempo, esta visión ubica al ser humano en una relación de horizontalidad con respecto al resto de la naturaleza, y no de dominación. Por tanto, en general, no prevalece en ellos la racionalidad de maximizar beneficios a costa de degradar la naturaleza (Toledo y Barrera-Bassols, 2008; Van der Ploeg, 2010). Los agricultores de origen boliviano cuyos casos son estudiados en el presente trabajo, pueden haber llegado a esta región con una cosmovisión de esta naturaleza, pero a medida que se adaptan a su nuevo contexto, pueden permearse en diferente grado de las ideas dominantes, en un proceso que genera una situación intermedia entre los dos tipos de ideologías (García, 2orrb). 
La metodología de indicadores resultó útil para transformar el concepto complejo y amplio de agrobiodiversidad de acuerdo con valores claros y de fácil interpretación. Así fue posible analizar y comparar las prácticas agrícolas de diferentes grupos de agricultores, y sus efectos sobre las diferentes dimensiones de la agrobiodiversidad. Sin embargo, se consideró que, para analizar y comparar conocimientos y percepciones, fue conveniente utilizar otras metodologías de tipo cualitativo como la entrevista en profundidad, que permitió ahondar en las temáticas estudiadas y comprender los resultados obtenidos (Minayo, 2012; Miles et al., 2013). Es así como ambas metodologías resultaron complementarias, primero, con la simplificación de la información, para que luego se pudiera entender su complejidad.

El presente trabajo comprobó que a aquellos agricultores que han adoptado el sistema de producción bajo invernadero en el Cinturón Hortícola Platense corresponden manejos y visiones que tienden a disminuir la agrobiodiversidad, en comparación con aquellos que todavía cultivan al aire libre. Además, dentro de estos grupos existen variaciones que se asocian con sus orígenes y con conocimientos ancestrales que hacen que los agricultores de origen boliviano promuevan una mayor biodiversidad en comparación con los de origen europeo. En este sentido, existen diferencias entre las percepciones de los personajes estudiados, así como en la cultura; disconformidades que actuaron como filtros para que algunos agricultores resistieran a las presiones externas.

\section{Conclusiones}

El presente trabajo busca ser un aporte al entendimiento de cómo un modelo de producción o determinados cambios tecnológicos ocurridos en el territorio tienen impactos significativos en los procesos de cambio y desarrollo sustentable de las áreas rurales desde aspectos ecológicos, sociales y económicos.

Se considera que para conservar la agrobiodiversidad y avanzar hacia sistemas sustentables, es necesario cambiar el concepto de la agricultura como una 'fábrica de productos’ para adoptar otro socialmente más justo, económicamente viable y ecológicamente adecuado, como el que proponen varias culturas tradicionales. Para ello se deberá modificar la visión que se propone desde los ámbitos científicosacadémicos, del mercado, de agricultores y principalmente del Estado, ya que puede fomentar condiciones que sustentan la creación de estrategias de desarrollo territorial sustentables. Así se podría dar respuesta a problemas específicos que generan situaciones de crecimiento económico, cohesión social y sostenibilidad ambiental. 


\section{Agradecimientos}

Agradecemos a los agricultores que dedicaron su valioso tiempo a participar del trabajo. Asimismo, al Consejo Nacional de Investigaciones Científicas y Técnicas (Conicet) por la beca doctoral otorgada a la primera autora.

\section{Referencias}

Abbona, E. A., Sarandón, S. J., Marasas, M. E. y Astier, M. (2007). Ecological sustainability evaluation of traditional management in different vineyard systems in Berisso, Argentina. Agriculture, Ecosystems and Environment, 119(3-4), 335- 345.

Acevedo-Osorio, A. y Angarita Leiton, A. (2013). Metodología para la evaluación de sustentabilidad, a partir de indicadores locales para el diseño y desarrollo de programas agroecológicos - Mesilpa. Bogotá: Corporación Universitaria Minuto de Dios.

Altieri, M. Á. y Nicholls, C. I. (2007). Biodiversidad y manejo de plagas en agroecosistemas. Vol. 2. Barcelona: Icaria Editorial.

Ander-Egg, E. (2000). Métodos y técnicas de investigación social. Buenos Aires: Lumen-Humanitas.

Astier, M., Speelman, E. N., López-Ridaura, S., Masera, O. R. y Gonzalez-Esquivel, C. E. (20II). Sustainability indicators, alternative strategies and trade-offs in peasant agroecosystems: analysing 15 case studies from Latin America. International Journal of Agricultural Sustainability, 9(3), 409-422.

Bédard, L. (2015). El ecologismo de la cosmovisión andina. Tinkuy boletín de Investigación y Debate, 22, II6-125. Recuperado de http://littlm.umontreal.ca/fileadmin/Documents/ FAS/litterature_langue_moderne/Documents/2-Recherche/Tinkuy_22_2015.pdf Blandi, M. L. (2016). Tecnología del invernáculo en el Cinturón Hortícola Platense: análisis de la sustentabilidad y los factores que condicionan su adopción por parte de los productores (Tesis doctoral). Facultad de Ciencias Agrarias y Forestales - UNLP, La Plata, Argentina. Recuperado de http://hdl.handle.net/10915/52015

Blandi, M. L., Sarandón, S. J., Flores, C. C. y Veiga, I. (2015). Evaluación de la sustentabilidad de la incorporación del cultivo bajo cubierta en la horticultura platense. Revista de la Facultad de Agronomía de La Plata, II4(2), 25I-264.

Blesh, M. y Barret, G.W. (2006). Farmer's attitudes regarding agrolandscape ecology: a regional comparison. Journal of sustainable agriculture, 28(3), I2I-I43.

Bonicatto, M. M., Marasas, M. E., Pochettino, M. L. y Sarandón, S. J. (20I4). La semilla en la conservación de los gustos y la historia. 
Leisa, 30(4). Recuperado de http://www.leisa-al.org/web/index.php/ volumen-30-numero-4/I078-la-semilla-en-la-conservacion-de-los-gustos-y-la-historia Bunnell, F. L. (2008). Indicators for sustaining biological diversity in Canada's most controversial forest type. Coastal temperate rainforest. Ecological indicators, 8(2), I49-157.

Cababié, J., Bonicatto, M. M. y Abbona, E. (2015). Semillas y saberes de los agricultores familiares. ¿Cuál es el rol de las ferias de intercambio en su reproducción y conservación? Revista Facultad de Agronomía, II4(Núm. Esp. I), I22-128.

Eisenhardt, K. M. (1989). Building Theories from Case Study Research. Academy of Management Review, I4(4), 532-550.

Eisenhardt, K. M. (1991). Better stories and better constructs: the case for rigor and comparative logic. Academy of Management Review, I6(3), 620-627.

Flores, C. C. (2012). Evaluación de la sustentabilidad de un proceso de transición agroecológica en sistemas de producción hortícolas familiares del partido de La Plata. (Tesis de Maestría). Facultad de Ciencias Agrarias y Forestales - UNLP, La Plata, Argentina.

García, M. (20Ira). El cinturón hortícola platense: ahogándonos en un mar de plásticos. Un ensayo acerca de la tecnología, el ambiente y la política. Theomai, 23 (Primer semeste 20II), 35-53.

García, M. (20Ib). Proceso de acumulación de capital en campesinos. El caso de los horticultores bolivianos de Buenos Aires (Argentina). Cuadernos de desarrollo rural, 8(66), $47-70$.

García, M. (2014). Crítica al enfoque clásico de innovación tecnológica: Estudio de caso del invernáculo en el Cinturón Hortícola Platense. Geograficando, ro(r). Recuperado de http://www.geograficando.fahce.unlp.edu.ar/article/view/GEOvionozaor

Gargoloff, N. A., Abonna, E. y Sarandón, S. J. (20ro). Análisis de la racionalidad ecológica en agricultores hortícolas de La Plata, Argentina. Revista brasileira de agroecologia, 5(2), 288-302.

Garrido-Fernandez, F. E. (2006). Los agricultores como actores de la política agroambiental. Un enfoque multidimensional. Papers: Revista de sociología, 8I, 37-62.

Gliessman, S. R. (20I4). Agroecology: the ecology of sustainable food systems. Boca Raton: CRC press.

Guzmán Casado, G. I. y Alonso Mielgo, A .M. (2007). La investigación participativa en Agroecología: una herramienta para el desarrollo sustentable. Ecosistemas, I6(I), 24-36. Hang, G., Kebat, C., Bravo, M. L., Larrañaga, G., Seibane, C., Ferraris, G., Otaño, M. y Blanco, V. (2010). Identificación de sistemas de producción hortícola en el partido de La Plata, provincia de Buenos Aires, Argentina. Bioagro, 22(I), 8I-86. 
Kaiser-Bunbury, C. N., Mougal, J., Whittington, A. E., Valentin, T., Gabriel, R., Olesen, J. M. y Blüthgen, N. (in press). Ecosystem restoration strengthens pollination network resilience and function. Nature, 542(7640), 223-227.

Marasas, M., Blandi, M. L., Dubrovsky Berensztein, N. y Fernandez, V. (2014). Transición agroecológica de sistemas convencionales de producción a sistemas de base ecológica. Características, criterios y estrategias. En S. J. Sarandón y C. C. Flores (Eds.), Agroecología: bases teóricas para el diseño y manejo de agroecosistemas sustentables (pp. 4II-436). La Plata: Editorial de la Universidad de La Plata.

Marradi, A., Archentti, N. y Piovani, J. (2007). Metodología de las ciencias sociales. Buenos Aires: Emecé.

Miles, M. B., Huberman, A. M. y Saldana, J. (2013). Qualitative data analysis. Temple: Sage. Minayo, M. C. (20I2). Análise qualitativa: teoria, passos e fidedignidade. Ciência $\mathcal{E}$ Saúde Coletiva, I7(3), 62I-626.

Minayo, M. C. S. y Minayo-Gómez, C. (2003). Difíceis e possíveis relações entre métodos quantitativos e qualitativos nos estudos de problemas de saúde. En P. Goldenberg, R. M. Giffoni Marsiglia y M. H. de Andréa Gomes (Orgs.), O Clássico e o Novo (pp. I17-I42). Brasil: Editora Fiocruz.

Michell, J. (1983). Case and situation analysis. The Sociological Review, 3I(2), I87-2II.

Nahed, T. J. (2008). Aspectos metodológicos en la evaluación de la sostenibilidad de sistemas agrosilvopastoriles. Avances en investigación agropecuaria, I2(3), 3-19.

Nicholls, C. (2006). Bases agroecológicas para diseñar e implementar una estrategia de manejo de hábitat para control biológico de plagas. Agroecología, I, 37-48.

Nicholls, C. I. y Altieri, M. A. (20I2). Modelos ecológicos y resilientes de producción agrícola para el siglo XXI. Agroecología, 6, 28-37.

Peres, F., Costa, M. J., Meneses, R. K., Lerner, R. y Claudio, L. (2007). El Uso de Pesticidas en la Agricultura y la Salud del Trabajador Rural en Brasil. Ciencia Eo Trabajo, 9(26),158-163.

Pérez, M. (2010). Horticultura de base ecológica en el cordón bonaerense sur. Una aproximación desde sus prácticas. (Tesis de maestría). Universidad Nacional de La Plata - FCAyF, La Plata, Argentina.

Sabogal Aguilar, J. y Hurtado, E. (2008). Elementos del concepto racionalidad ambiental. Revista de la facultad de ciencias económicas: investigación y reflexión. Nueva Granada - Colombia, I6(2),II7-I32.

Sarandón, S. J. (2009). Biodiversidad, agrobiodiversidad y agricultura sustentable: Análisis del Convenio sobre Diversidad Biológica. En T. L. Siccard y M.A. Altieri 
(Eds.), Vertientes del pensamiento agroecológico: fundamentos y aplicaciones. Ideas $2 \mathrm{I}$ (pp. 105-130). Bogotá: Sociedad Científica Latinoamericana de Agroecología (Socla).

Sarandón, S. J., Flores, C. C., Gargoloff, N. A. y Blandi, M. L. (20I4). Análisis y evaluación de agroecosistemas: construcción y aplicación de indicadores. En S. J. Sarandón y C. C. Flores (Eds.), Agroecología. Bases teóricas para el diseño y manejo de agroecosistemas sustentables (pp. 375-410). Recuperado de http://hdl.handle. net/10915/37280

Selis, D. (20I2). Análisis de la institucionalidad asociada a los procesos de innovación tecnológica en el sector hortícola del Gran La Plata. Mundo Agrario, I2(24). Recuperado de http://www.mundoagrario.unlp.edu.ar/index.php/mundoagrario/ article/view/vi2n24aII/2I49

Soares Schenkel, M. D. G., Costabeber, J. A., da Silveira, P.R. y Schenkel, C.A. (2007). A formação e a conversão de grupos de agricultores agroecológicos no alto uruguai catarinense e gaúcho. Revista Brasileira de Agroecologia, 2(I). Recuperado de http://coral.ufsm.br/desenvolvimentorural/textos/agroecologia\%202004\%204.pdf

Stupino, S., Iermanó, M. J., Gargoloff, N. A. y Bonicatto, M. M. (2014). La biodiversidad en los agroecosistemas. En S. J. Sarandón y C. C. Flores (Eds.), Agroecología. Bases teóricas para el diseño y manejo de agroecosistemas sustentables (pp. I3I-158). Recuperado de http://hdl.handle.net/10915/37280

Taylor, S. J., Bogdan, R. y DeVault, M. (2015). Introduction to qualitative research methods: A guidebook and resource. New Jersey: John Wiley \& Sons.

Toledo, V. M. (2005). La memoria tradicional: la importancia agroecológica de los saberes locales. Leisa, 20(4), 16-19.

Toledo, V. M. y Barrera-Bassols, N. (2008). La memoria biocultural: la importancia ecológica de las sabidurías tradicionales Barcelona: Icaria.

Toledo Machado, A. (2007). Biodiversidade e agroecologia. En W. S. de Boef, M. H. Thijssen, J. B. Ogliari y B. R. Sthapit. Biodiversidade e agricultores. Fortalecendo o manejo comunitário, 2(pp. 40-45). Brasil: Organizaçao de Porto Alegre.

UNEP (2000). Apéndice. En The Biodiversity Agenda. Decisiones adoptadas por la conferencia de las partes en el convenio sobre la diversidad biológica en su quinta reunión. Agenda concretada en Nairobi.

Van der Ploeg, J. D. (2010). Nuevos campesinos. Campesinos e imperios alimentarios. Icaria: Barcelona.

Yin, R. (2013). Case study research: Design and methods. 5ta edición. London: Sage. 